\title{
Top-Down Control of Human Visual Cortex by Frontal and Parietal Cortex in Anticipatory Visual Spatial Attention
}

\author{
Steven L. Bressler, ${ }^{1,2}$ Wei Tang, ${ }^{1}$ Chad M. Sylvester, ${ }^{3}$ Gordon L. Shulman, ${ }^{4}$ and Maurizio Corbetta ${ }^{3,4,5}$ \\ ${ }^{1}$ Center for Complex Systems and Brain Sciences and ${ }^{2}$ Department of Psychology, Florida Atlantic University, Boca Raton, Florida 33431, and Departments \\ of ${ }^{3}$ Radiology, ${ }^{4}$ Neurology, and ${ }^{5}$ Anatomy and Neurobiology, Washington University School of Medicine, St. Louis, Missouri 63110
}

Advance information about an impending stimulus facilitates its subsequent identification and ensuing behavioral responses. This facilitation is thought to be mediated by top-down control signals from frontal and parietal cortex that modulate sensory cortical activity. Here we show, using Granger causality measures on blood oxygen level-dependent time series, that frontal eye field (FEF) and intraparietal sulcus (IPS) activity predicts visual occipital activity before an expected visual stimulus. Top-down levels of Granger causality from FEF and IPS to visual occipital cortex were significantly greater than both bottom-up and mean cortex-wide levels in all individual subjects and the group. In the group and most individual subjects, Granger causality was significantly greater from FEF to IPS than from IPS to FEF, and significantly greater from both FEF and IPS to intermediate-tier than lower-tier ventral visual areas. Moreover, top-down Granger causality from right IPS to intermediate-tier areas was predictive of correct behavioral performance. These results suggest that FEF and IPS modulate visual occipital cortex, and FEF modulates IPS, in relation to visual attention. The current approach may prove advantageous for the investigation of interregional directed influences in other human brain functions.

Key words: cerebral cortex; fMRI; vision; attention; Granger causality; frontal cortex; parietal cortex

\section{Introduction}

A strong theoretical foundation supports the concept of topdown modulation of sensory processes by control regions of the cerebral cortex (Desimone and Duncan, 1995; Kastner and Ungerleider, 2000; Corbetta and Shulman, 2002). Anticipatory visual spatial attention, whereby an observer voluntarily attends the visual field location of an impending target, improves target detection and behavioral performance (Eriksen and Hoffman, 1972; Bashinski and Bacharach, 1980; Posner, 1980; Tong, 2003). Human functional magnetic resonance imaging (fMRI) studies have shown that anticipatory visual spatial attention involves stimulus-independent changes in blood oxygen level-dependent (BOLD) signals from frontal, parietal, and visual cortical regions (Corbetta and Shulman, 2002; Serences and Yantis, 2006), but have not shown directed influences between these regions. Evidence that transcranial magnetic stimulation (TMS) of human frontal (Ruff et al., 2006) and parietal (Ruff et al., 2008) regions affects visual cortical BOLD activity, and that electrical microstimulation of monkey frontal cortex affects visual cortical electrical activity (Moore and Armstrong, 2003), indicates that pathways exist

Received April 23, 2008; revised Aug. 29, 2008; accepted Aug. 30, 2008.

This work was supported by the J. S. McDonnell Foundation, the National Institute of Neurological Disorders and Stroke (Grants F30 NS057926-01 and R01 NS48013), the National Institute of Mental Health (Grant R01 MH7192006), and the Marie Curie Chair European Union (Grant MEXC-CT-2004-006783). Granger causality analysis was based on the Econometrics Toolbox of James P. LeSage.

Correspondence should be addressed to Steven L. Bressler, Center for Complex Systems and Brain Sciences, Florida Atlantic University, 777 Glades Road, Boca Raton, FL 33431. E-mail: bressler@fau.edu.

D0I:10.1523/JNEUROSCI.1776-08.2008

Copyright $\odot 2008$ Society for Neuroscience ～0270-6474/08/2810056-06\$15.00/0 for top-down modulation of visual cortex and that stimulation of these pathways can produce attention-like behavioral effects, but does not show a relation between top-down modulation and visual attention. Here we present evidence suggesting that visual cortex receives top-down modulation from frontal and parietal areas in relation to visual attention.

Directed influences between BOLD signals were measured by Granger causality (Granger, 1969; Roebroeck et al., 2005), which quantifies the improvement in predicting one brain region's signal that results from inclusion of another region's signal in that prediction. Although Granger causality cannot prove that neuronal communication occurs between regions, it can nonetheless provide supporting evidence for it. Several properties of Granger causality make it suitable for the study of interregional directed influences in human cognition. First, it is measured from ongoing activity in subjects performing a cognitive task and, thus, does not depend on nonphysiological intervention as in TMS or microstimulation. Second, it is an asymmetric measure that allows quantification of predictability in both directions between regions, unlike symmetric measures such as cross-correlation or mutual information. Third, being based on predictability, it provides stronger evidence for neuronal communication than do measures simply showing temporal precedence of one time series over another. Finally, it can measure directed influences at a specific task-related time.

Granger causality was evaluated on BOLD time series in frontal eye field (FEF), intraparietal sulcus (IPS), and visual occipital regions immediately preceding visual target presentation, when attentional spatial selectivity was maximal (Sylvester et al., 2007). 


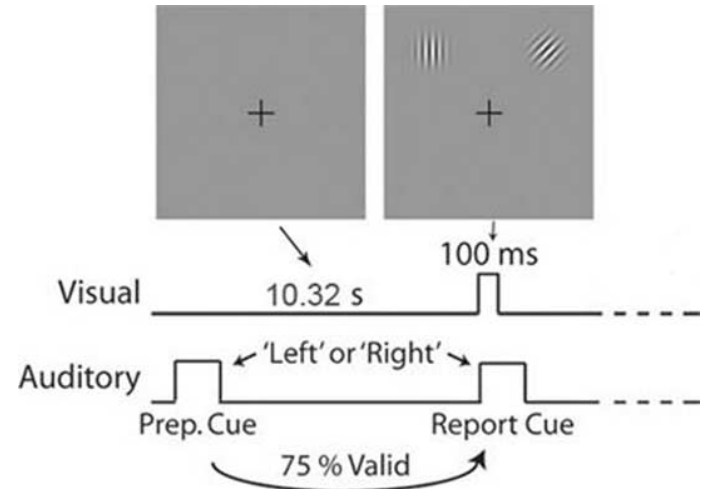

Data

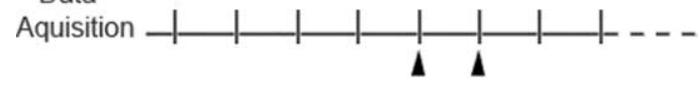

Figure 1. Visual spatial attention behavioral paradigm. An auditory preparatory cue ("Left" or "Right") began each trial, instructing subjects to attend a location left or right of the vertical meridian. After a stimulus-onset asynchrony (10.32 s), visual targets appeared for $100 \mathrm{~ms}$ centered at both locations, concurrent with an auditory report cue ("Left" or "Right"). BOLD data were acquired with $T R=2.064 \mathrm{~s}$. The sample times used for analysis are marked by triangles. The figure is not drawn to scale.

Top-down Granger causality was significantly greater than both bottom-up and mean cortex-wide levels. Granger causality was also significantly greater from FEF to IPS than from IPS to FEF, and from FEF and IPS to intermediate-tier ventral visual areas than to low-tier areas. Furthermore, top-down Granger causality from the right intraparietal sulcal area to intermediate-tier areas predicted correct behavioral performance. These results imply that top-down control was in effect before the target in this task.

\section{Materials and Methods}

Participants and task. Six right-handed subjects (three male, three female), aged 26-30, performed a demanding visual-spatial attention task (Sylvester et al., 2007) (Fig. 1). Subjects had no history of neurological illness, and had normal or corrected-to-normal vision. Informed consent was obtained as per human studies committee guidelines at Washington University School of Medicine. Each subject performed 1400-1600 trials over 16-24 scanning sessions. Eye position was monitored to ensure fixation on a central crosshair. Trials began with a $500 \mathrm{~ms}$ preparatory cue (the spoken word "left" or "right"), which directed attention to one of two locations at $5^{\circ}$ eccentricity in the upper hemifield, $45^{\circ}$ clockwise or counterclockwise from the vertical meridian (Fig. 1). Right- and leftcued trials were randomly intermixed with equal probability. After a stimulus-onset asynchrony (SOA) of 6.192 (25\%), 8.256 (25\%), or 10.32 s (50\%), visual target stimuli appeared for $100 \mathrm{~ms}$ centered at both locations, concurrent with an auditory report cue ("left" or "right"). Targets were 3.5 cycle per degree Gabor patches $\left(0.3^{\circ}\right.$ Gaussian envelope SD). On valid trials $(75 \%)$, the report cue matched the preparatory cue. Subjects indicated the orientation (left tilt, vertical, right tilt) of the report-cued patch by pressing one of three buttons. High (50\%) and low (5-12\%) contrast targets were presented in separate scans. Stimulus parameters were adjusted based on in-scanner practice sessions to yield approximately $70 \%$ correct valid trial performance. Valid trials having the 10.32 s SOA, with either target contrast level and cue direction, were included in the analysis. The selective analysis of trials with $10.32 \mathrm{~s}$ SOA was aimed at maximizing anticipatory effects, which were shown by Sylvester et al. (2007) to increase with time after the cue. Mean task performance was $70.6 \%$ correct for valid and $61.5 \%$ correct for invalid trials (chance $=$ $33.3 \%$ ), indicating that subjects used the preparatory cue to discriminate the target.

Data acquisition. BOLD data were acquired with a Siemens Allegra 3T scanner using an asymmetric spin-echo echoplanar sequence [retention time $(\mathrm{TR})=2.064 \mathrm{~s}$, echo time $=25 \mathrm{~ms}$, flip angle $=90^{\circ}, 32$ contiguous
$4 \mathrm{~mm}$ axial slices, $4 \times 4 \mathrm{~mm}$ in-plane resolution). BOLD images were motion-corrected within and between runs, corrected for across-slice timing differences, resampled into $3 \mathrm{~mm}$ isotropic voxels, and warped into a standardized atlas space.

Region of interest creation. For each subject, BOLD data at each voxel were subjected to a general linear model using in-house software. Constant and linear terms over BOLD runs modeled baseline and linear drift. Sine waves modeled low-frequency noise $(<0.009 \mathrm{~Hz})$. Separate $\delta$ function regressors coded each time point after the preparatory cue. To generate individual-trial time series, modeled responses of the appropriate event type were summed with the residuals from the linear model at each time point.

Regions of interest (ROIs) outside retinotopic cortex were created by voxel-wise ANOVA in each subject over the first six trial time points using the residuals dataset. An in-house clustering algorithm defined ROIs from the map of the main effect of cue direction. ROIs were $8 \mathrm{~mm}$ spheres centered on map peaks with $z$-scores $>3$; spheres within $12 \mathrm{~mm}$ of each other were consolidated into a single ROI. ROIs were retained for subsequent analysis if they were present in at least 6 of the 12 subject hemispheres so as to reduce intersubject variability, which could come from variability in BOLD signal strength, task strategy (causing the relative involvement of different functional areas to vary), or localization of functional areas.

If a subject lacked a particular ROI, the $z$ threshold was lowered to 2; if the subject still lacked the ROI, it was not included in subsequent analyses. This procedure yielded ROIs in FEF (at the junction of the superior frontal sulcus and the precentral sulcus), anterior IPS (aIPS), and posterior IPS (pIPS).

To create ROIs inside retinotopic cortical areas, subjects first passively viewed contrast-reversing checkerboard stimuli extending along the horizontal and vertical meridians. From a contrast of responses to the horizontal and vertical meridians, early visual region borders were handdrawn on flattened representations of each subject's anatomy using the Caret software suite (Van Essen et al., 2001). The V3A ROI consisted of $\mathrm{V} 3 \mathrm{~A}$ voxels with responses that varied with the direction of the preparatory cue. In separate localizer scans, subjects passively viewed highcontrast $(\sim 50 \%)$ Gabor patches flickering at $4 \mathrm{~Hz}$. In each $12 \mathrm{~s}$ block, a patch randomly appeared at one of five locations (two target, two mirrored across the horizontal meridian, and one central). Voxels representing each of these locations were localized by $t$ tests: voxel responses to each non-central stimulus were significantly larger than those to its mirror stimulus across the vertical meridian; responses to the central location $\left(1^{\circ}\right.$ width) were significantly larger than the summed responses to all other locations. Subdivisions of early visual cortex (V1v, V2v, VP, V4) were made from the conjunction of voxels with a stimulus preference in the localizer scans and the retinotopic regions for upper hemifield locations.

BOLD time series preprocessing. Outlier voxels were rejected, based on their BOLD amplitude variability over trials, using Tukey's boxplot technique. BOLD time series were $z$-normalized to have zero mean and unit variance.

Granger causality method. Granger causality testing followed the method of Greene (2002). For any two voxels, $X$ and $Y$, Granger causality is tested from $X$ to $Y$, and from $Y$ to $X$. For $X$ to $Y$, two different linear regression models are considered. The restricted model is: $Y(t)=$ $\alpha_{1} Y(t-m)+\varepsilon_{1}(t)$, where $Y(t)$ is the $Y$ time series at time $t, Y(t-m)$ is the $m$-lagged $Y$ time series, $\alpha_{1}$ is the regression coefficient, and $\varepsilon_{1}(t)$ is the restricted model residual at time $t$.

The unrestricted model is: $Y(t)=\alpha_{2} Y(t-m)+\beta X(t-m)+\varepsilon_{2}(t)$, where $Y(t)$ is the $Y$ time series at time $t, Y(t-m)$ and $X(t-m)$ are the $m$-lagged time series of $Y$ and $X, \alpha_{2}$ and $\beta$ are regression coefficients, and $\varepsilon_{2}(t)$ is the unrestricted model residual at time $t$.

Granger causality significance testing. If the variability of the residual of the unrestricted model is significantly reduced compared with that of the restricted model, then there is an improvement in the prediction of $Y$ due to $X$. The amount of reduction is measured as an $F$ statistic: $F=\left[\left(R S S_{\mathrm{r}}-\right.\right.$ $\left.\left.R S S_{\mathrm{ur}}\right) / m\right] /\left[R S S_{\mathrm{ur}} /(T-2 m-1)\right]$, where $R S S_{\mathrm{r}}$ is the restricted residual sum of squares, $R S S_{\mathrm{ur}}$ is the unrestricted residual sum of squares, $m$ is the lag value, and $T$ is the total number of observations used to estimate the 
unrestricted model. The $F$ statistic approximately follows an $F$ distribution with degrees of freedom $m$ and $(T-2 m-1)$. If the $F$ statistic from $X$ to $Y$ is significant (i.e., greater than the critical value at $p<0.05$ in the standard $F_{\mathrm{m}, \mathrm{T}}-$ $2 \mathrm{~m}-1$ distribution), then the unrestricted model yields a better explanation of $Y(t)$ than does the restricted model, and $X$ is said to Granger cause $Y$.

Granger causality BOLD data analysis. For a given ROI pair, $F$ statistics were computed in both directions between BOLD time series of every voxel pair. The portion of voxel pairs carrying Granger causality was quantified for each ROI pair in both directions as the fraction of $F$ statistics that were significant $(p<0.05)$. To compare significant fractions of $F$ distributions having equal numbers of voxel pairs, the $\mathrm{McNe}$ -

mar (1947) statistic was calculated. Significance of this statistic came from comparison with the standard $\chi^{2}$ distribution with one degree of freedom. F-statistic distributions were compared by the Mann-Whitney $U$ test (Mann and Whitney, 1947) when the distributions had unequal numbers. Correction for multiple comparisons was by Dunn's procedure (Kirk, 1982).

Granger causality was tested on $F$ statistics derived from linear regression models using the last two BOLD measurements of the anticipatory period (marked by triangles in Fig. 1). Balanced numbers of correct and incorrect validly cued trials were used to maximize the number of trials while avoiding biases that might otherwise result. Frontal and parietal ROIs consisted of spatially selective voxels in right and left FEF, aIPS, and pIPS. Visual occipital ROIs consisted of voxels in right and left V1v, V2v, $\mathrm{V} 3 \mathrm{~A}, \mathrm{VP}$, and V4. With the exception of two subjects, who lacked significantly identifiable ROIs in left FEF and aIPS, 60 pairwise frontoparietaloccipital relationships (six frontal and parietal with 10 visual occipital ROIs) were examined, as were 4 pairwise frontal-parietal relationships (two FEF with two IPS, anterior and posterior combined). Each ROI pair was tested independently of all others.

$F$-statistic distributions were derived for individual subjects. These distributions were either analyzed separately for each subject or were combined across subjects for group analysis. Analyses of the veridical data were compared with analyses of randomized data sets created in two ways. First, trial-randomized data consisted of ROIs having the same voxels as the veridical data, and with $F$-statistic distributions computed with randomized trial order for each voxel. Trial-randomized data provided an estimate of expected Granger causality with physiological influences removed. Second, voxel-randomized data consisted of ROIs having voxels randomly selected from the entire cortex, and with $F$-statistic distributions computed with the same trial order for each voxel as the veridical data. Voxel-randomized data provided an estimate of Granger causality expected for any two randomly selected cortical locations.

\section{Results}

Granger causality was tested for every voxel pair in each of the 60 frontoparietal-occipital ROI pairs in top-down (from FEF or IPS to visual occipital) and bottom-up (from visual occipital to FEF or IPS) directions. F-statistic distributions (Fig. 2) contained significant fractions, indicating Granger causality, in both directions for every ROI pair in the veridical, as well as in the voxelrandomized and trial-randomized data. The fact that a variable fraction of the voxel pairs comprising an ROI pair had significant $F$-statistics indicated that the ROIs were not spatially homogeneous in their influences on one another. Top-down Granger causality in the veridical data was significantly greater $(p<$ 0.00001 ) in all six subjects than in the voxel-randomized or trialrandomized data. In contrast, bottom-up Granger causality in the veridical data was lower than in the voxel-randomized data in all subjects [significantly lower $(p<0.005)$ in three subjects], but was significantly greater $(p<0.05)$ than in the trial-randomized data in all subjects. Veridical top-down Granger causality was significantly greater than bottom-up Granger causality $(p<1.0$ $\times 10^{-30}$ ) in all subjects (Fig. $3 \mathrm{~A}$ ) and was significantly greater $(p<0.05)$ for 59 of 60 ROI pairs in a group analysis (Fig. $3 C$ ). These results suggest top-down modulation of visual occipital areas by FEF and IPS.

Granger causality was also tested in both directions for every voxel pair in each of the four frontal-parietal ROI pairs. Veridical Granger causality from FEF to IPS was significantly greater $\left(p<1.0 \times 10^{-30}\right)$ than that from IPS to FEF in the group and in five subjects (Fig. $3 B$ ). Granger causality from FEF to IPS in the veridical data was significantly greater $(p<$ $1.0 \times 10^{-30}$ ) than that in the voxel-randomized data in the group and in five subjects, and significantly greater $(p<1.0 \times$ $\left.10^{-30}\right)$ than that in the trial-randomized data in all subjects. Veridical Granger causality from IPS to FEF was significantly less $\left(p<1.0 \times 10^{-30}\right)$ than that in the voxel-randomized data in the group and in four subjects, and was significantly greater $\left(p<1.0 \times 10^{-30}\right)$ than that in the trial-randomized data in all subjects. Equivalent comparisons in both the voxelrandomized (Fig. 3D) and trial-randomized (Fig. 3E) data did not show any significant directional difference. Thus, FEF modulated IPS, and both regions modulated visual occipital cortex.

We next sought to determine whether top-down modulation was greater to some visual occipital regions than to others. In a group analysis, top-down Granger causality from frontal and parietal (left and right FEF, aIPS, and pIPS) regions to intermediate-tier ventral visual occipital regions (VP and V4) was significantly greater $\left(p<1.0 \times 10^{-40}\right)$ than that to low-tier regions (V1 and V2) of both the left and right hemispheres. In individual analyses, the effect was significant $(p<0.05)$ in five subjects for low-tier regions of the left hemisphere, and in four subjects for those of the right hemisphere. Equivalent comparisons on the voxel-randomized and trial-randomized data were not significant.

We then tested whether top-down modulation of visual cortex predicted correct responses to subsequent targets by comparing top-down Granger causality before correct and incorrect performance from each frontal and parietal ROI to all visual occipital ROIs combined. In a group analysis, significant $(p<0.05)$ performance differences were observed for each frontal and parietal ROI except the left aIPS. In individual subject analyses, a consistent performance difference in five subjects was only seen for right aIPS, with Granger causality significantly greater $(p<0.05)$ before correct than before incorrect performance. No consistent 


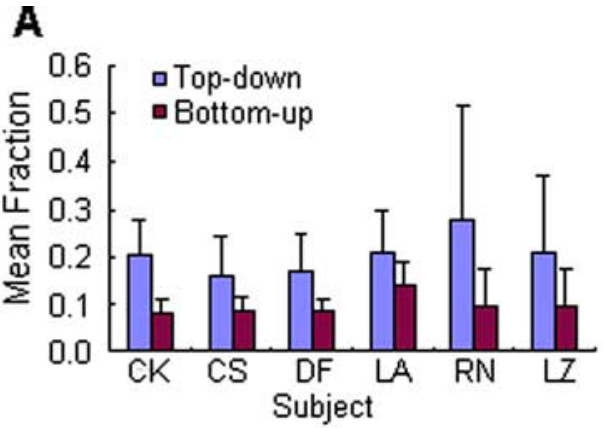

\section{B}

C
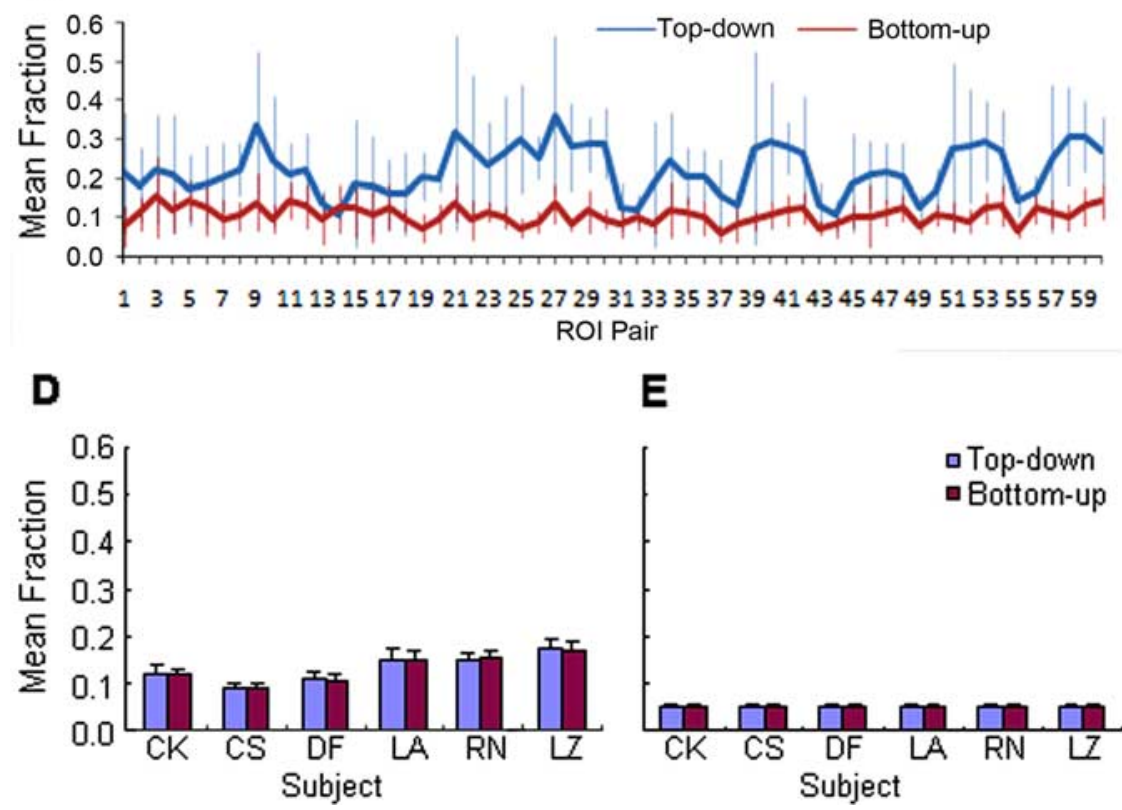

E

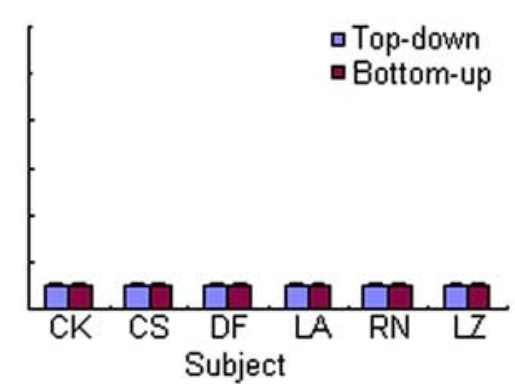

Figure 3. $A-E$, Top-down versus bottom-up Granger causality. The mean fraction of significant $F$ is significantly greater for top-down (blue) than for bottom-up (red) Granger causality when measured separately for each subject $(\boldsymbol{A})$ and for 59 of $60 \mathrm{ROI}$ pairs with all subjects combined $(\boldsymbol{C})$, but not in the voxel-randomized $(\boldsymbol{D})$ or trial-randomized $(\boldsymbol{E})$ data. Error bars indicate variability across $\mathrm{RO}$ pairs $(\boldsymbol{A}, \boldsymbol{B}, \boldsymbol{D}, \boldsymbol{E})$ or across subjects $(\boldsymbol{C})$. $\boldsymbol{B}$, The mean fraction of significant $F$ is significantly greater from FEF to IPS than from IPS to FEF for five of six subjects. C, ROI pairs follow left-to-right, top-to-bottom ordering in Figure $4 \mathrm{~A}$.

A

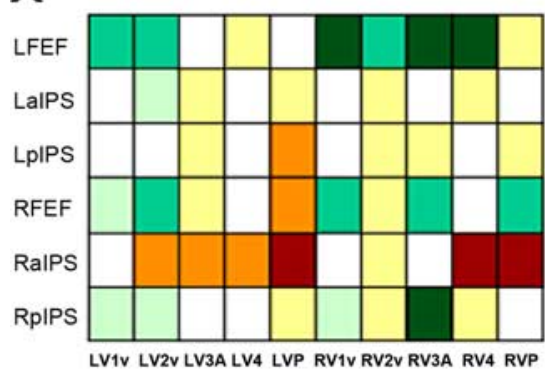

B

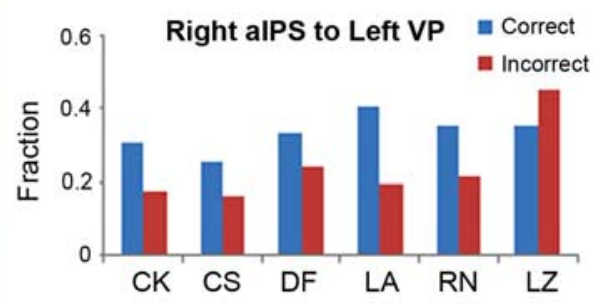

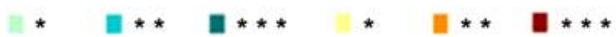

Fraction: correct < incorrect Fraction: correct $>$ incorrect

Figure 4. Top-down Granger causality before correct versus incorrect performance. $\boldsymbol{A}$, Grid specifying the significance of correct versus incorrect performance difference in a group analysis of top-down Granger causality: ${ }^{*} p<0.05,{ }^{* *} p<1.0 \times$ $10^{-10}{ }^{* * *} p<1.0 \times 10^{-30}$; white, not significant. Each cell represents Granger causality from the row-labeled ROI to the column-labeled ROI. $\boldsymbol{B}$, The fraction of significant top-down Granger causality from right alPS to left VP was significantly greater before correct (blue) than incorrect (red) performance in five of six subjects.

performance difference was observed for FEF-to-IPS Granger causality.

Top-down Granger causality for each of the frontoparietaloccipital and frontal-parietal ROI pairs was also separately com- pared for performance differences. In a group analysis, top-down Granger causality was significantly different $(p<0.05)$ before correct and incorrect performance for a number of ROI pairs (Fig. 4A). However, top-down Granger causality was significantly different $(p<0.05)$ consistently in five of six subjects only from right aIPS to left VP (Fig. $4 B$ ), being significantly greater $(p<0.05)$ before correct than before incorrect performance. In addition, top-down Granger causality from right aIPS was significantly greater $(p<0.05)$ prior to correct than incorrect performance to right and left V4 in four of six subjects, and to right VP in three of six subjects. These performance-related results could not be attributed to the greater magnitude of top-down Granger causality to intermediate-tier regions. No consistent across-subject performance differences were observed for the other ROI pairs in the top-down direction, for any pair in the bottom-up direction, or for any pair in either direction in either the voxelrandomized or trial-randomized data. The variability in ROI pairs showing significant differences in top-down Granger causality before correct and incorrect performance suggests that some top-down influences promote correct behavioral performance, whereas others may actually impair performance.

\section{Discussion}

The results of this study support the view that FEF and IPS exert top-down modulatory influences on visual cortex in relation to visual attention (Desimone and Duncan, 1995; Kastner and Ungerleider, 2000; Corbetta and Shulman, 2002; Moore et al., 2003; Serences and Yantis, 2006). Although studies using TMS of human FEF (Ruff et al., 2006) and IPS (Ruff et al., 2008), and electrical microstimulation of monkey FEF (Moore and Armstrong, 2003), demonstrate that pathways exist to carry influences from FEF and IPS to visual cortex, and stimulation of these pathways can produce behavioral effects similar to those produced by attention, they do not indicate whether top-down influences are exerted when subjects attend to a location under physiological conditions. Moreover, because stimulation is only applied to frontal or parietal cortex, these studies do not show any asymmetry between top-down and bottom-up influences. Our study reveals such an asymmetry by demonstrating that BOLD activity in FEF and IPS predicts BOLD activity in the visual cortex of subjects engaged in a visual attention task at levels significantly higher than bottom-up levels. Furthermore, top-down, 
but not bottom-up, predictability was significantly higher than the mean cortex-wide level. Such top-down predictability between BOLD signals may depend on the entrainment of slow neuroelectric excitability waves observed in visual attention (Lakatos et al., 2008).

The analysis of directed influences between FEF and IPS suggests that FEF modulates IPS far more than IPS modulates FEF during visual attention. To our knowledge, directed influences between frontal and parietal regions have not previously been investigated, although neural synchrony between them has been observed during attention (Buschman and Miller, 2007). Future analysis will consider the question of whether FEF-to-occipital Granger causality is direct or can be attributed to an influence through IPS, and likewise, whether IPS-to-occipital Granger causality can be attributed to FEF.

The finding of stronger top-down modulation of intermediate-tier areas (VP, V4) than of low-tier areas (V1, $\mathrm{V} 2$ ) is consistent with the known distribution of attentionrelated modulation in extrastriate cortex (Motter, 1993; Chelazzi et al., 1995; Luck et al., 1997; Mehta et al., 2000; Schroeder et al., 2001; Treue, 2001; Kastner and Pinsk, 2004). Similarly, our finding that top-down Granger causality from the right anterior IPS region to bilateral VP and V4 predicts subsequent task performance suggests that these areas may be critical nodes in attentional regulation of visual cortex. This finding is consistent with the theory that right parietal cortex influences spatial processing of both visual hemifields (Heilman et al., 1985; Mesulam, 1999; Siman-Tov et al., 2007), results showing that TMS of right IPS produces stimulusindependent BOLD changes in both left and right visual cortex (Ruff et al., 2008), and findings that task-related decreases in the BOLD signal of right anterior IPS and bilateral visual cortical regions correlate with behavioral improvement in visual perceptual learning (Mukai et al., 2007).

Our approach is based on a unique combination of experimental and analytic methods allowing measurement of eventrelated Granger causality in human BOLD data on a relatively short time scale. It bears a superficial resemblance to techniques measuring relative delay time between ROIs in eventrelated BOLD data, such as the mutual information-based method of Fuhrmann Alpert et al. (2007). The pairwise measure in that study is symmetric and is derived from longduration averaged BOLD time series. In contrast, our method provides asymmetric directed measures between ROIs, and is derived from short-duration single-trial BOLD time series. Although our study examined Granger causality at a fixed time point and time lag between ROIs, our method can also yield latency and relative delay information, as did Fuhrmann Alpert et al. (2007).

This study examined Granger causality at a single sample time during the anticipatory period. More comprehensive future studies of the same data set will involve analysis of other time samples, and will examine whether Granger causality can distinguish cortical representations of cued from uncued visual quadrants.

Granger causality is a measure of statistical relation and, thus, cannot identify the pathways carrying the effects observed in this study. It is possible that influences from a region other than FEF or IPS were responsible for those effects. However, as a whole, the set of comparisons comprising this study establish that significant unidirectional predictive relations exist from FEF and IPS to visual occipital cortex, and support the view that FEF and IPS exert top-down control of visual occipital processing in visual attention. Granger causality analysis of event-related BOLD data may prove to be a generally useful tool to noninvasively measure the causal interplay between different regions of the human brain in relation to cognition and emotion.

\section{References}

Bashinski HS, Bacharach VR (1980) Enhancement of perceptual sensitivity as the result of selectively attending to spatial locations. Percept Psychophys 28:241-248.

Buschman TJ, Miller EK (2007) Top-down versus bottom-up control of attention in the prefrontal and posterior parietal cortices. Science 315:1860-1862.

Chelazzi L, Biscaldi M, Corbetta M, Peru A, Tassinari G, Berlucchi G (1995) Oculomotor activity and visual spatial attention. Behav Brain Res 71:81-88.

Corbetta M, Shulman GL (2002) Control of goal-directed and stimulusdriven attention in the brain. Nat Rev Neurosci 3:201-215.

Desimone R, Duncan J (1995) Neural mechanisms of selective visual attention. Annu Rev Neurosci 18:193-222.

Eriksen CW, Hoffman JE (1972) Temporal and spatial characteristics of selective encoding from visual displays. Percept Psychophys 12:201-204.

Fuhrmann Alpert G, Sun FT, Handwerker D, D’Esposito M, Knight RT (2007) Spatio-temporal information analysis of event-related BOLD responses. Neuroimage 15:1545-1561.

Granger CWJ (1969) Investigating causal relations by econometric methods and cross-spectral methods. Econometrica 34:424-438.

Greene WH (2002) Econometric analysis, Ed 5. Upper Saddle River, NJ: Prentice-Hall.

Heilman KM, Watson RT, Valenstein E (1985) Neglect and related disorders. In: Clinical neuropsychology (Heilman KM, Valenstein E, eds), pp 243-293. New York: Oxford UP.

Kastner S, Pinsk MA (2004) Visual attention as a multilevel selection process. Cogn Affect Behav Neurosci 4:483-500.

Kastner S, Ungerleider LG (2000) Mechanisms of visual attention in the human cortex. Annu Rev Neurosci 23:315-341.

Kirk RE (1982) Experimental design: procedures for the behavioral sciences. Belmont, CA: Wadsworth.

Lakatos P, Karmos G, Mehta AD, Ulbert I, Schroeder CE (2008) Entrainment of neuronal oscillations as a mechanism of attentional selection. Science 320:110-113.

Luck SJ, Chelazzi L, Hillyard SA, Desimone R (1997) Neural mechanisms of spatial selective attention in areas V1, V2, and V4 of macaque visual cortex. J Neurophysiol 77:24-42.

Mann HB, Whitney DR (1947) On a test of whether one of two random variables is stochastically larger than the other. Ann Math Stat 18:50-60.

McNemar Q (1947) Note on the sampling error of the difference between correlated proportions or percentages. Psychometrika 12:153-157.

Mehta AD, Ulbert I, Schroeder CE (2000) Intermodal selective attention in monkeys. I: distribution and timing of effects across visual areas. Cereb Cortex 10:343-358.

Mesulam MM (1999) Spatial attention and neglect: parietal, frontal and cingulate contributions to the mental representation and attentional targeting of salient extrapersonal events. Philos Trans R Soc Lond B Biol Sci 354:1325-1346.

Moore T, Armstrong KM (2003) Selective gating of visual signals by microstimulation of frontal cortex. Nature 421:370-373.

Moore T, Armstrong KM, Fallah M (2003) Visuomotor origins of covert spatial attention. Neuron 40:671-683.

Motter BC (1993) Focal attention produces spatially selective processing in visual cortical areas V1, V2, and V4 in the presence of competing stimuli. J Neurophysiol 70:909-919.

Mukai I, Kim D, Fukunaga M, Japee S, Marrett S, Ungerleider LG (2007) Activations in visual and attention-related areas predict and correlate with the degree of perceptual learning. J Neurosci 27:11401-11411.

Posner MI (1980) Orienting of attention. Q J Exp Psychol 32:3-25.

Roebroeck A, Formisano E, Goebel R (2005) Mapping directed influence over the brain using Granger causality and fMRI. Neuroimage 25:230-242. 
Ruff CC, Blankenburg F, Bjoertomt O, Bestmann S, Freeman E, Haynes JD, Rees G, Josephs O, Deichmann R, Driver J (2006) Concurrent TMSfMRI and psychophysics reveal frontal influences on human retinotopic visual cortex. Curr Biol 16:1479-1488.

Ruff CC, Bestmann S, Blankenburg F, Bjoertomt O, Josephs O, Weiskopf N, Deichmann R, Driver J (2008) Distinct causal influences of parietal versus frontal areas on human visual cortex: evidence from concurrent TMSfMRI. Cereb Cortex 18:817-827.

Schroeder CE, Mehta AD, Foxe JJ (2001) Determinants and mechanisms of attentional modulation of neural processing. Front Biosci 6:D672-D684.

Serences JT, Yantis S (2006) Selective visual attention and perceptual coherence. Trends Cogn Sci 10:38-45.

Siman-Tov T, Mendelsohn A, Schonberg T, Avidan G, Podlipsky I, Pessoa L,
Gadoth N, Ungerleider LG, Hendler T (2007) Bihemispheric leftward bias in a visuospatial attention-related network. J Neurosci 27:11271-11278.

Sylvester CM, Shulman GL, Jack AI, Corbetta M (2007) Asymmetry of anticipatory activity in visual cortex predicts the locus of attention and perception. J Neurosci 27:14424-14433.

Tong F (2003) Primary visual cortex and visual awareness. Nat Rev Neurosci 4:443-459.

Treue S (2001) Neural correlates of attention in primate visual cortex. Trends Neurosci 24:295-300.

Van Essen DC, Drury HA, Dickson J, Harwell J, Hanlon D, Anderson CH (2001) An integrated software suite for surface-based analyses of cerebral cortex. J Am Med Inform Assoc 8:443-459. 